\title{
THE METHOD OF ANALYTICAL REGULARIZATION IN THE ELECTROMAGNETIC WAVE SCATTERING BY THIN DISKS
}

\author{
M.V. Balaban ${ }^{*}$, A.I. Nosich ${ }^{*}$, A. Altintas ${ }^{\dagger}$, T.M. Benson ${ }^{\dagger \dagger}$ \\ *Institute of Radiophysics and Electronics NASU, U1. Proskury, 12, Kharkov 61085, Ukraine \\ ${ }^{\dagger}$ Bilkent University, Electrical \& Electronics Engineering Department, Bilkent, 06800-Ankara, Turkey \\ ${ }^{++}$George Green Institute for Electromagnetics Research, University of Nottingham, Nottingham NG7 2RD, UK
}

Keywords: diffraction, disk, analytical regularization.

\begin{abstract}
We consider the problem of diffraction of an arbitrary electromagnetic wave by a thin disk made from different materials and located in free space. Here we imply a zerothickness perfectly electrically conducting (PEC) disk, and also thin electrically resistive (ER) and dielectric disks whose thickness is much smaller than the disk radius and the free space wavelength, and also much smaller than the skin-layer depth in the ER disk case. The method used for the modeling is based on the integral equation (IE) technique and analytical regularization. Starting with Maxwell's equations, boundary conditions and the radiation condition at infinity we obtain a set of coupled dual IEs (DIEs) for the unknowns and then reduce this set of equations to the coupled IEs of the Fredholm second kind. To verify our results we calculate the far field characteristics in the case of the PEC disk with the incident field being the field of horizontal electrical dipole located on the disk axis.
\end{abstract}

\section{Introduction}

The problem of electromagnetic field scattering by a thin disk has been interested in since long ago. This is explained by many different applications of this canonical shape. Besides of traditional applications in the printed disk antennas with PEC or ER disks, thin dielectric disk is used as a simplified model of the tree leave [1]. Still besides, thin few-micronradius disks are met as resonators of semiconductor lasers with ultralow thresholds [2]. Many methods of computational electromagnetics have been used in their analysis, starting from empirical methods and finishing with the method of moments (MoM) and finite difference time domain method (FDTD). However, the use of these methods leads to difficulties such as prohibitively large matrices and impossibility of estimation of the error of calculations. In contrast, by using the method of IEs with analytical regularization it is possible to escape these difficulties.

\section{Problem statement}

We consider the problem of diffraction of a given timeharmonic electromagnetic field by a zero-thickness dielectric disk. The geometry of this problem is illustrated in Figure 1.

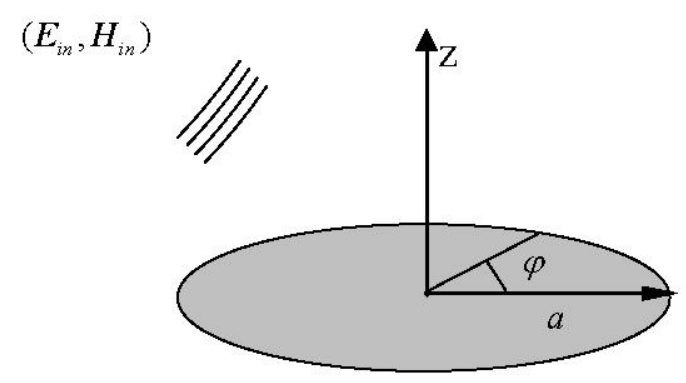

Figure 1. Disk geometry

The disk of the radius $a$ is located in free space at the plane $(z=0)$. We introduce the cylindrical coordinates $(r, \varphi, z)$ with the origin on the axis of symmetry of the disk and denote total field as a sum of the fields scattered by the disk and the incident one:

$$
E=E_{s c}+E_{\text {in }}, \quad H=H_{s c}+H_{\text {in }}
$$

The components of the scattered field must satisfy the set of homogeneous Maxwell equations outside the disk, the 3-D radiation condition, the condition of local integrability of power (edge condition), and the generalized boundary conditions (see [3]) on the disk surface, at $(z=0, r<a)$ :

$$
\begin{aligned}
& \frac{1}{2}\left[E_{t g}^{+}+E_{t g}^{-}\right]=Z_{0} R \cdot \vec{n} \times\left[H_{t g}^{+}-H_{t g}^{-}\right], \\
& \frac{Z_{0}}{2}\left[H_{t g}^{+}+H_{t g}^{-}\right]=-S \cdot \vec{n} \times\left[E_{t g}^{+}-E_{t g}^{-}\right] .
\end{aligned}
$$

Here, $Z_{0}$ is the free-space impedance, and $R$ and $S$ are the electric and magnetic resistivities. For a thin dielectric disk they are given by

$$
\begin{array}{lll}
R=\frac{i Z}{2} \cot \left(\frac{\sqrt{\varepsilon_{r} \mu_{r}} k \tau}{2}\right), & \left|\varepsilon_{r} \mu_{r}\right| \gg 1, & \tau \ll \lambda_{0} . \\
S=\frac{i}{2 Z} \cot \left(\frac{\sqrt{\varepsilon_{r} \mu_{r}} k \tau}{2}\right), & \left|\varepsilon_{r} \mu_{r}\right| \gg 1, & \tau \ll \lambda_{0} .
\end{array}
$$


Here, $Z$ is the relative impedance of the disk material, $k=\omega / c$ is the wavenumber, $\varepsilon_{r}$ is the relative permittivity, $\mu_{r}$ is the relative conductivity, $\lambda_{0}$ is the wavelength in free space, and $\tau$ is the thickness of the disk.

For a thin electrically resistive (ER) disk they are given by

$$
R=\frac{1}{Z_{0} \sigma \tau}, \quad \sigma / \omega \gg 1, \quad S=\infty .
$$

For a zero-thickness PEC disk they are given by

$$
R=0, \quad S=\infty
$$

On the rest part of the plane $(z=0)$ the components of the field are continuous.

\section{Basic equations}

We express the scattered field components normal to the disk in terms of the azimuth-angle Fourier series and the radial inverse integral Hankel transform:

$$
E_{s c, z}^{ \pm}=\sum_{m=-\infty}^{\infty} e^{i m \varphi} \int_{0}^{\infty} e^{ \pm i \gamma(\kappa) \zeta} e_{m, z}^{ \pm}(\kappa) J_{|m|}(\kappa \rho) \kappa d \kappa
$$

$$
Z_{0} H_{s c, z}^{ \pm}=\sum_{m=-\infty}^{\infty} e^{i m \varphi} \int_{0}^{\infty} e^{ \pm i \gamma(\kappa) \zeta} h_{m, z}^{ \pm}(\kappa) J_{|m|}(\kappa \rho) \kappa d \kappa
$$

where $e_{m, z}^{ \pm}(\kappa)$ and $h_{m, z}^{ \pm}(\kappa)$ are the images of the normal to the disk field components in the spectral domain. They are the unknown functions to be found. Then the tangential to the disk components can be written as follows:

$$
\begin{gathered}
\left(\begin{array}{c}
E_{s c, r}^{ \pm} \\
-i E_{s c, \varphi}^{ \pm}
\end{array}\right)=\sum_{m=-\infty}^{\infty} e^{i m \varphi} \cdot \int_{0}^{\infty} e^{ \pm i \gamma(\kappa) \zeta} \bar{H}_{m}(\kappa \rho)\left(\begin{array}{c} 
\pm i \gamma(\kappa) e_{m, z}^{ \pm}(\kappa) \\
-k a h_{m, z}^{ \pm}(\kappa)
\end{array}\right) d \kappa(9) \\
\left(\begin{array}{c}
Z_{0} H_{s c, r}^{ \pm} \\
-i Z_{0} H_{s c, \varphi}^{ \pm}
\end{array}\right)=\sum_{m=-\infty}^{\infty} e^{i m \varphi} \cdot \int_{0}^{\infty} e^{ \pm i \gamma(\kappa) \zeta} \bar{H}_{m}(\kappa \rho)\left(\begin{array}{c} 
\pm i \gamma(\kappa) h_{m, z}^{ \pm}(\kappa) \\
-k a e_{m, z}^{ \pm}(\kappa)
\end{array}\right) d \kappa(10)
\end{gathered}
$$

Here

$$
\bar{H}_{m}(\kappa \rho)=\left(\begin{array}{cc}
J_{|m|}^{\prime}(\kappa \rho) & m J_{|m|}(\kappa \rho) /(\kappa \rho) \\
m J_{|m|}(\kappa \rho) /(\kappa \rho) & J_{|m|}^{\prime}(\kappa \rho)
\end{array}\right)
$$

is the kernel matrix function of the vector Hankel transform, $\rho=r / a, \zeta=z / a$ and $\gamma(\kappa)=\sqrt{(k a)^{2}-\kappa^{2}}$.

On substituting (9) and (10) to (2) and continuity conditions outside the disk, we, in general case, obtain the set of coupled DIEs for the four unknown functions in the spectral domain:

$$
\begin{aligned}
& \begin{cases}\int_{0}^{\infty} \bar{H}_{m}(\kappa \rho)\left(\begin{array}{l}
i \gamma(\kappa)\left(u_{m}^{-}(\kappa)+e^{i \gamma(\kappa)\left|\zeta_{0}\right|} u_{m}^{\zeta_{0},-}(\kappa)\right)+2 R i k a u_{m}^{-}(\kappa) \\
-k a\left(v_{m}^{+}(\kappa)+e^{i \gamma(\kappa)\left|\zeta_{0}\right|} v_{m}^{\zeta_{0},+}(\kappa)\right)-2 R \gamma(\kappa) v_{m}^{+}(\kappa)
\end{array}\right) d \kappa=\left(\begin{array}{l}
0 \\
0
\end{array}\right) & \rho<1, \\
\int_{0}^{\infty} \bar{H}_{m}(\kappa \rho)\left(\begin{array}{c}
i k a u_{m}^{-}(\kappa) \\
-\gamma(\kappa) v_{m}^{+}(\kappa)
\end{array}\right) d \kappa=\left(\begin{array}{l}
0 \\
0
\end{array}\right) & \rho>1,\end{cases} \\
& \begin{cases}\int_{0}^{\infty} \bar{H}_{m}(\kappa \rho)\left(\begin{array}{l}
i \gamma(\kappa)\left(v_{m}^{-}(\kappa)+\left.e^{i \gamma(\kappa)\left|\zeta_{0}\right|}\right|_{m} ^{\zeta_{0},-}(\kappa)\right)+2 S i k a v_{m}^{-}(\kappa) \\
k a\left(u_{m}^{+}(\kappa)+e^{i \gamma(\kappa)\left|\zeta_{0}\right|} u_{m}^{\zeta_{0},+}(\kappa)\right)+2 S \gamma(\kappa) u_{m}^{+}(\kappa)
\end{array}\right) d \kappa=\left(\begin{array}{l}
0 \\
0
\end{array}\right) & \rho<1, \\
\int_{0}^{\infty} \bar{H}_{m}(\kappa \rho)\left(\begin{array}{c}
i k a v_{m}^{-}(\kappa) \\
\gamma(\kappa) u_{m}^{+}(\kappa)
\end{array}\right) d \kappa=\left(\begin{array}{l}
0 \\
0
\end{array}\right) & \rho>1,\end{cases}
\end{aligned}
$$

Here $u_{m}^{ \pm}(\kappa)$ и $v_{m}^{ \pm}(\kappa)$ are linear combination of original unknown image function:

$$
\begin{aligned}
& u_{m}^{ \pm}(\kappa)=\left(e_{m, z}^{+}(\kappa) \pm e_{m, z}^{-}(\kappa)\right) / 2 \\
& v_{m}^{ \pm}(\kappa)=\left(h_{m, z}^{+}(\kappa) \pm h_{m, z}^{-}(\kappa)\right) / 2
\end{aligned}
$$

The obtained set of DIEs can be written in the following abstract form:

$$
\begin{aligned}
\int_{0}^{\infty} \bar{H}_{m}(\kappa \rho) & \cdot \bar{S}_{1}(k a, \kappa, \rho) \cdot X(\kappa) d \kappa \\
= & \int_{0}^{\infty} \bar{H}_{m}(\kappa \rho) \cdot \bar{S}_{2}(k a, \kappa, \rho) \cdot X_{0}(\kappa) d \kappa
\end{aligned}
$$

where $\bar{S}_{1,2}(k a, \kappa, \rho)$ are singular diagonal matrix-functions of two variables with the singular point at infinity $(\kappa=\infty)$, $X(\kappa)$ is the unknown vector-function, $X_{0}(\kappa)$ is the given vector-function. Note that the singularity of $\bar{S}_{2}(k a, \kappa, \rho)$ leads to the limitation of $X_{0}(\kappa)$ in terms of the allowed class 
of functions. To solve (15), we use the method of analytical regularization [4]. It consists of the following two parts:

First, we split the function $\bar{S}_{1}(k a, \kappa, \rho)$ into the singular and regular parts:

$$
\bar{S}_{1}(k a, \kappa, \rho)=\bar{\Sigma}_{1}(k a, \kappa, \rho)+\overline{\mathrm{P}}_{1}(k a, \kappa, \rho)
$$

Then (15) can be rewritten as follows:

$$
\int_{0}^{\infty} \bar{H}_{m}(\kappa \rho) \cdot \bar{\Sigma}_{1}(k a, \kappa, \rho) \cdot X(\kappa) d \kappa=F(\rho, X(\ldots))
$$

where the right-hand part is

$$
\begin{gathered}
F(\rho, X(\ldots))=\int_{0}^{\infty} \bar{H}_{m}(\kappa \rho) \cdot\left(-\overline{\mathrm{P}}_{1}(k a, \kappa, \rho) \cdot X(\kappa)+\right. \\
\left.+\bar{S}_{2}(k a, \kappa, \rho) \cdot X_{0}(\kappa)\right) d \kappa
\end{gathered}
$$

The solution of "canonical" IE is sought in the following form:

$$
X(\lambda)=\int_{0}^{\infty} \bar{K}_{m}(\lambda, \rho) \cdot F(\rho, X(\ldots)) d \rho
$$

Now we substitute expression (18) into (19) and reduce the coupled DIEs (15) to the following Fredholm IE of the second kind:

$$
\begin{array}{r}
X(\lambda)+\int_{0}^{\infty} \bar{K}_{m}(\lambda, \rho) \cdot \int_{0}^{\infty} \bar{H}_{m}(\kappa \rho) \cdot \overline{\mathrm{P}}_{1}(k a, \kappa, \rho) \cdot X(\kappa) d \kappa d \rho \\
=\int_{0}^{\infty} \bar{K}_{m}(\lambda, \rho) \cdot \int_{0}^{\infty} \bar{H}_{m}(\kappa \rho) \cdot \bar{S}_{2}(k a, \kappa, \rho) \cdot X_{0}(\kappa) d \kappa d \rho
\end{array}
$$

Extraction of the singular part of integral operators in (12), (13) leads to two different-type DIEs in the case of PEC ( $R=0$ and $S=\infty$ ) and dielectric or ER disks. Finding the solutions in both cases with $(m \neq 0)$ is based on decupling the DIEs by the integration in $\rho$ and introduction of four (in general case) constants of integration. This procedure leads to the DIEs, which can be cast to the following short form:

$$
\begin{cases}\int_{0}^{\infty}\left\{\kappa^{\delta} u_{m}(\kappa)-f(\kappa)\right\} J_{m}(\kappa \rho) d \kappa=0 & (\rho<1) \\ \int_{0}^{\infty} u_{m}(\kappa) J_{m}(\kappa \rho) d \kappa=0 & (\rho>1)\end{cases}
$$

Such DIEs can be solved analytically by using the Abel integral transform and inverse Hankel integral transform in the case of $\delta= \pm 1$ or just inverse Hankel integral transform in the case of $\delta=0$. Additional equations for finding the constants of integration follow from the conditions of local integrability of power near the disk edge.

As an example, we show the final integral equation of the Fredholm second kind $(m \neq 0)$ in case when the diffraction by the PEC disk is considered.

$$
\begin{aligned}
& x_{m}(\lambda)-\int_{0}^{\infty} i w(\kappa) x_{m}(\kappa) K_{m}^{(1)}(\kappa, \lambda) d \kappa+i k a 2 m A_{m} I_{m}^{(1)} \lambda^{-1 / 2}(\lambda+1)^{-1} J_{m+3 / 2}(\lambda)+ \\
& \quad+i 2 m B_{m} I_{m}^{(2)} \lambda^{-1 / 2}(\lambda+1)^{-1} J_{m-1 / 2}(\lambda)=\int_{0}^{\infty} i \kappa^{-1} \gamma(\kappa)(\kappa+1)^{2} e^{i \gamma(\kappa)\left|\zeta_{0}\right|} x_{m}^{0}(\kappa) K_{m}^{(1)}(\kappa, \lambda) d \kappa \\
& y_{m}(\lambda)-\int_{0}^{\infty} w(\kappa) y_{m}(\kappa) \lambda \gamma^{-1}(\lambda) K_{m}^{(-1)}(\kappa, \lambda) d \kappa+2 m A_{m} I_{m}^{(3)} \lambda^{1 / 2} \gamma^{-1}(\lambda)(\lambda+1)^{-1} J_{m+1 / 2}(\lambda)+ \\
& \quad+2 m k a B_{m} I_{m}^{(4)} \lambda^{1 / 2}(\lambda+1)^{-1} J_{m-3 / 2}(\lambda)=-\int_{0}^{\infty} i(\kappa+1)^{2} e^{i \gamma(\kappa)\left|\zeta_{0}\right|} y_{m}^{0}(\kappa) \lambda \gamma^{-1}(\lambda) K_{m}^{(-1)}(\kappa, \lambda) d \kappa \\
& -\int_{0}^{\infty} i w(\kappa) x_{m}(\kappa) G_{m}^{(1)}(\kappa) d \kappa+i k a 2 m A_{m} I_{m}^{(1)}-i 2 m B_{m} I_{m}^{(2)}=\int_{0}^{\infty} i \kappa^{-1} \gamma(\kappa)(\kappa+1)^{2} e^{i \gamma(\kappa)\left|\zeta_{0}\right|} x_{m}^{0}(\kappa) G_{m}^{(1)}(\kappa) d \kappa \\
& -\int_{0}^{\infty} w(\kappa) y_{m}(\kappa) G_{m}^{(-1)}(\kappa) d \kappa+2 m A_{m} I_{m}^{(3)}-k a 2 m B_{m} I_{m}^{(4)}=-\int_{0}^{\infty} i(\kappa+1)^{2} e^{i \gamma(\kappa)\left|\zeta_{0}\right|} y_{m}^{0}(\kappa) G_{m}^{(-1)}(\kappa) d \kappa
\end{aligned}
$$

Here $x_{m}(\lambda), y_{m}(\lambda)$ are the unknown functions, $A_{m}, B_{m}$ are the unknown constants of integration, $K_{m}^{( \pm 1)}(\kappa, \lambda), G_{m}^{( \pm 1)}(\kappa)$ are the kernel functions, $w(\kappa)$ is the weight function, $x_{m}^{0}(\lambda)$ and $y_{m}^{0}(\lambda)$ are the given functions determined by the incident field, and $I_{m}^{(1,2,3,4)}$ are known coefficients. 


\section{Numerical results}

The Fredholm second kind nature of the derived equations guarantees the existence of solutions of (22) and the convergence of numerical algorithm based on any reasonable discretization scheme. For example, it can be a simple projection scheme with the basis functions taken as the socalled step functions:

$$
\delta_{n}^{s}(\lambda)= \begin{cases}1 & \lambda \in[s(n-1), s n) \\ 0 & \lambda \notin[s(n-1), s n)\end{cases}
$$

To verify the obtained equations we calculate the main characteristics in the case of the PEC disk with the incident field being the field of a horizontal electrical dipole located on the disk axis and oriented along the line $(\varphi=0)$. They are the far-field radiation patterns and the total radiated power. Besides, we calculate truncation and discretization errors in the computation of the total radiated power.

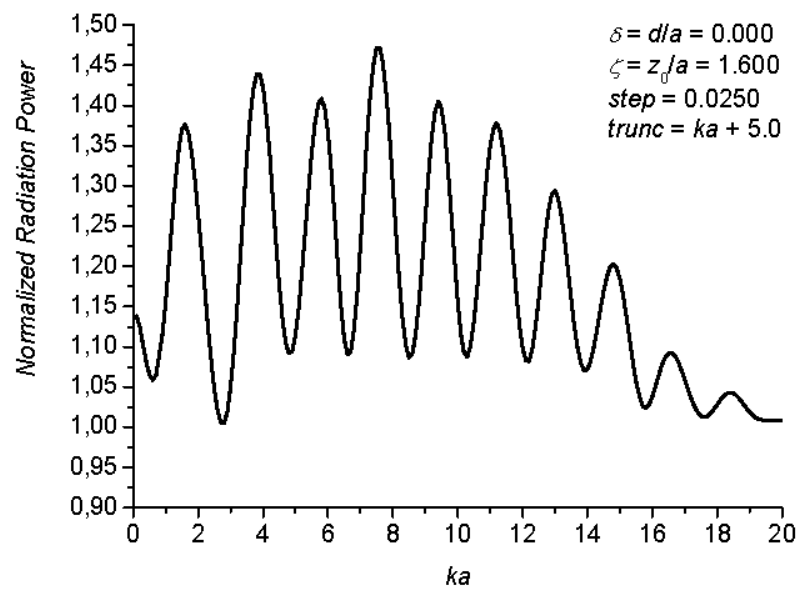

Figure 2a. Normalized radiation power vs. the normalized disk radius.

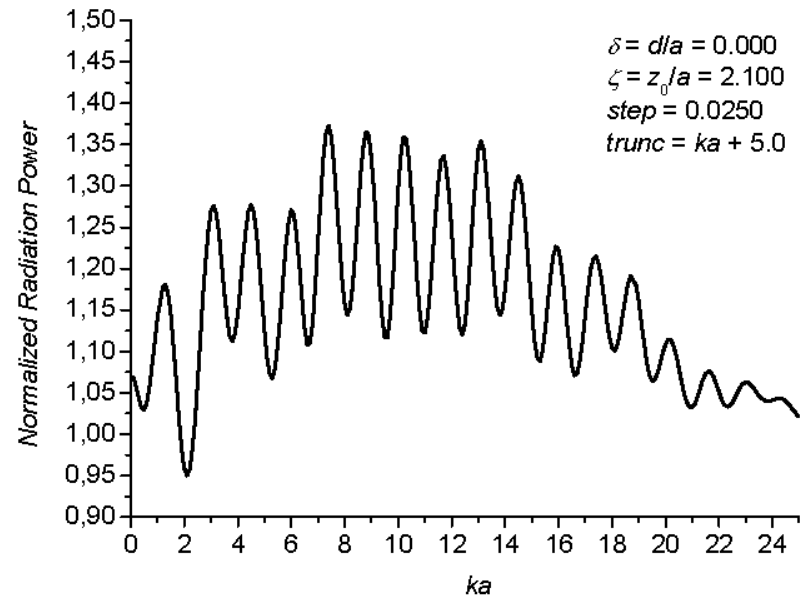

Figure $2 \mathrm{~b}$. Normalized radiation power vs. the normalized disk radius.
Figures $2 \mathrm{a}-2 \mathrm{~b}$ show the normalized radiation power vs. the frequency normalized by the disk radius. Here $\zeta$ is the normalized distance from the dipole to the disk center, trunc is the truncation value of the domain of integration in (22) that is adapted to the frequency as $k a+5$. Figures $3 a-3 b$ show the relative computation errors given by:

$$
E r_{\text {curr }}=\left|P_{\text {curr }}-P_{\text {prev }}\right| /\left|P_{\text {prev }}\right|
$$

where $P_{\text {prev }}$ is the normalized total power at the previous computation point, $P_{\text {curr }}$ is the normalized total power at the current point. Figure 4 shows the dependence of $P$ vs. $\zeta$, which is the normalized distance between disk and dipole. Figure 5 shows the far field radiation patterns for some characteristic values of parameters.

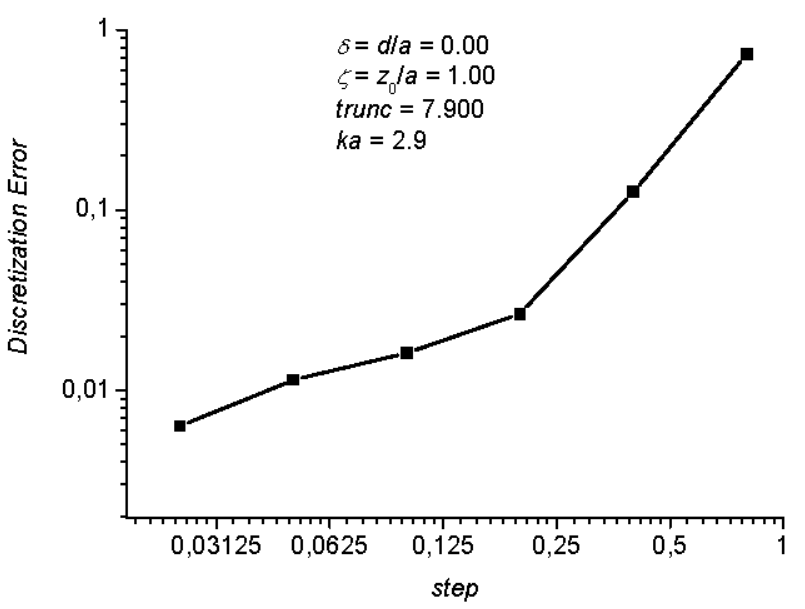

Figure 3a. Discretization error vs. the grid step.

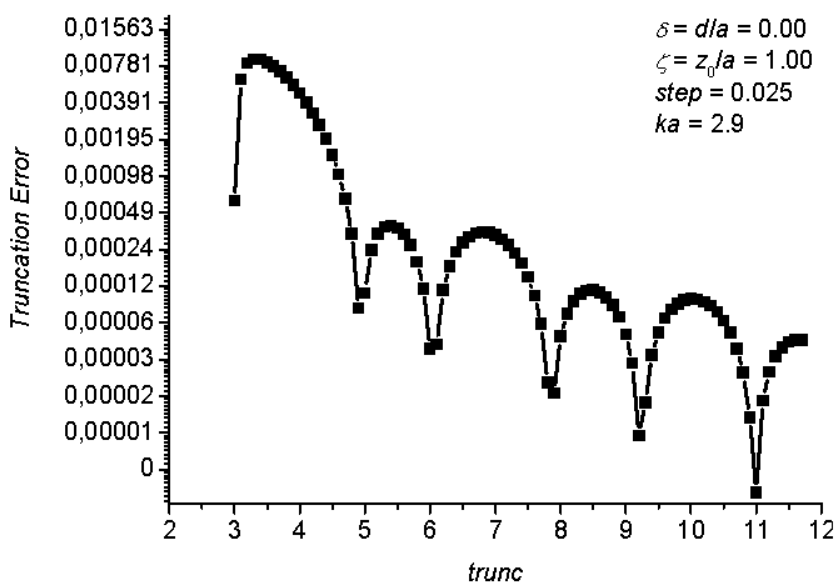

Figure $3 \mathrm{~b}$. The computation error vs. the value of truncation of the integration domain. 


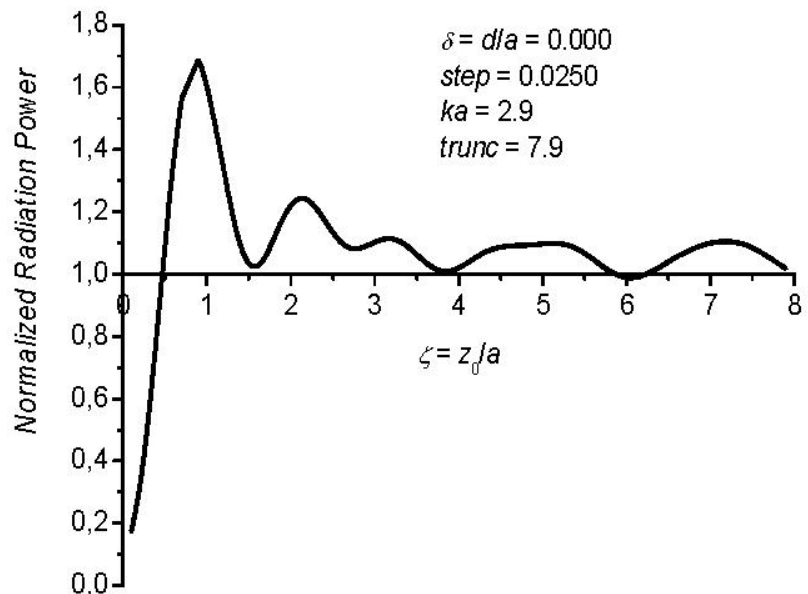

Figure 4. Normalized radiation power vs. the distance from the disk to the dipole.

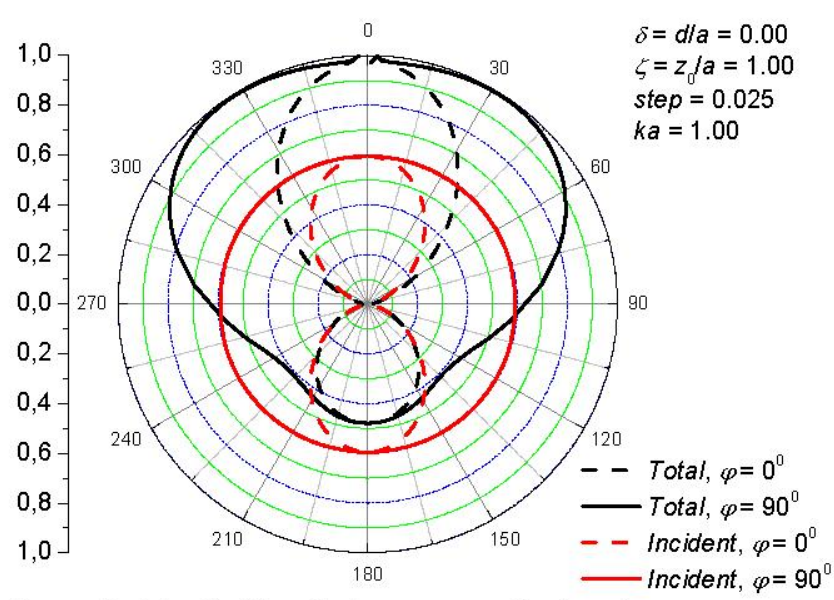

Figure 5a. Far field radiation pattern for $k a=1$.

\section{Acknowledgements}

This work was supported by the National Academy of Sciences of Ukraine via project PORIG $\# 36 / 07 \mathrm{H}$, the Turkish Council for Research in Science and Technology and the Royal Society, UK via joint projects.

\section{References}

[1] I.-S. Koh, K. Sarabandi, "A new approximate solution for scattering by thin dielectric disks of arbitrary size and shape" , IEEE Trans Antennas Propag., vol 53, pp. 1920-1926, 2005.

[2] X. Liu, W. Fang, Y. Huang, X. H. Wu, S. T. Ho, H. Cao, R. P. H. Chang, "Optically pumped ultraviolet microdisk laser on a silicon substrate", Applied Physics Letters, vol. 84, No 14, pp. 2488-2490, 2004.

[3] E. Bleszynski, M. Bleszynski, T. Jaroszewicz, "Surfaceintegral equations for electromagnetic scattering from impenetrable and penetrable sheets", IEEE Antennas Propag. Mag., vol. 35, pp. 14-24, 1993.

[4] A.I. Nosich, "Method of analytical regularization based on the static part inversion in wave scattering by

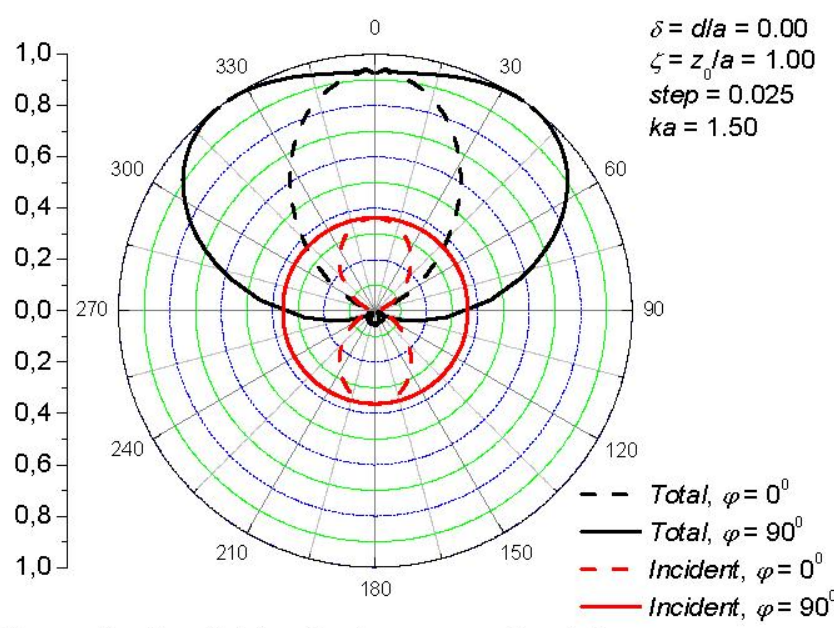

Figure $5 \mathrm{~b}$. Far field radiation pattern $k a=1.5$.

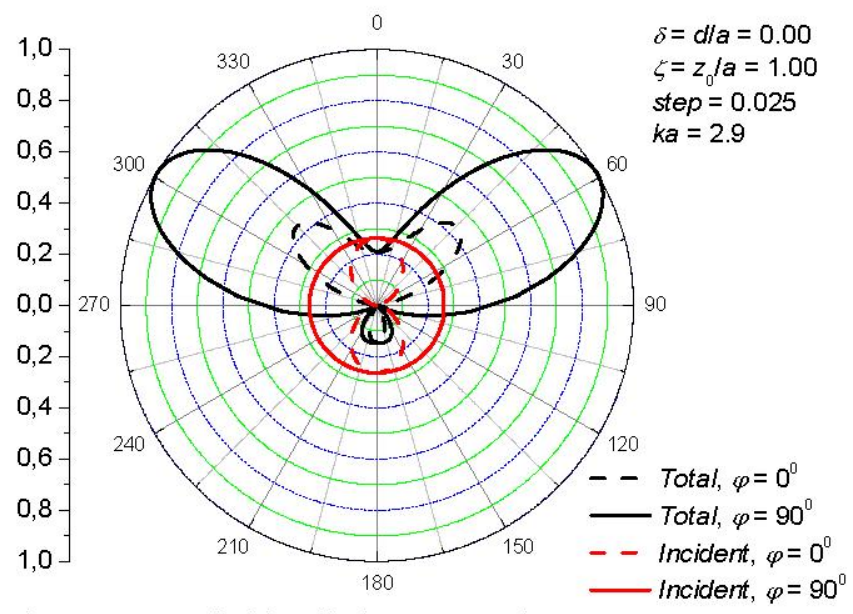

Figure $5 \mathrm{c}$. Far field radiation pattern $k a=2.9$.

imperfect thin screens", $J$. Telecommunications and Information Technology, Warsaw: NIT Press, 2001, no 3, pp. $72-79$. 